\title{
THE PROBABILITY OF CONTAINMENT FOR MULTITYPE BRANCHING PROCESS MODELS FOR EMERGING EPIDEMICS
}

\author{
SIMON E. F. SPENCER, ${ }^{*}$ University of Warwick \\ PHILIP D. O'NEILL, ${ }^{* *}$ University of Nottingham
}

\begin{abstract}
This paper is concerned with the definition and calculation of containment probabilities for emerging disease epidemics. A general multitype branching process is used to model an emerging infectious disease in a population of households. It is shown that the containment probability satisfies a certain fixed point equation which has a unique solution under certain conditions; the case of multiple solutions is also described. The extinction probability of the branching process is shown to be a special case of the containment probability. It is shown that Laplace transform ordering of the severity distributions of households in different epidemics yields an ordering on the containment probabilities. The results are illustrated with both standard epidemic models and a specific model for an emerging strain of influenza.
\end{abstract}

Keywords: Branching process; epidemic; influenza; stochastic epidemic

2010 Mathematics Subject Classification: Primary 92D30

Secondary $60 \mathrm{~J} 80$

\section{Introduction}

This paper is concerned with the definition and calculation of containment probabilities for stochastic epidemic models. Our approach involves defining a certain multitype branching process to describe the spread of disease in a specified population of individuals partitioned into households. The branching process itself is rather general in the sense that it can describe a wide range of underlying epidemic models. By supposing that individuals in the population have potentially infectious contacts with those in the 'outside world' according to specified Poisson processes, it is then possible to define a containment probability. The majority of the paper is then devoted to developing theory by which this containment probability can be calculated. We also present a result involving Laplace transform orderings by which different epidemics can be compared, and finish with some numerical examples. To begin with, the following paragraphs give a brief motivation for the problem at hand.

When a potentially pandemic disease first emerges, there may be an opportunity to use targeted interventions to eliminate the disease altogether, whilst the number of individuals infected remains small. For this to be achieved, the geographical spread of cases must be relatively localised, so that the population at risk of infection can be effectively targeted. Since no intervention strategy can completely prevent disease transmission, it may take some time

Received 28 September 2010; revision received 14 October 2010.

* Postal address: Department of Statistics, University of Warwick, Coventry CV4 7AL, UK.

Email address: s.e.f.spencer@warwick.ac.uk

** Postal address: School of Mathematical Sciences, University of Nottingham, University Park, Nottingham NG7 2RD, UK. Email address: philip.oneill@ nottingham.ac.uk 
for the intervention to eliminate the disease. It is vital that during this period the infection does not escape from the region to which the intervention has been applied to cause further outbreaks elsewhere. As outlined above, in this paper we describe such an emerging disease using a general model. Moreover, different intervention measures can be included in the model and the corresponding containment probabilities used to assess their effectiveness.

Although the idea of a containment probability appears previously in the literature [7], this paper is the first to attempt to develop a theoretical framework in order to calculate such a probability. Through this analytical approach we can view the containment probability as a generalisation of the extinction probability for a branching process. Traditionally, the effectiveness of intervention strategies has been measured by their effect on the reproduction number $R_{0}$, roughly defined as the expected number of infections caused by a typical infective in a fully susceptible population. If the intervention reduces $R_{0}$ to 1 or below then, in a large population, the epidemic will die out with probability close to 1 . Hence, $R_{0}$ is a threshold parameter. However, if the intervention fails to reduce $R_{0}$ to 1 , there is still some probability that the epidemic will die out - particularly if there are only a small number of cases when the intervention is applied. This can make $R_{0}$ more difficult to interpret than the probability of containment.

The remainder of this paper is structured as follows. In Section 2 we describe the multitype branching process model used and define a related reproduction number. In Section 3 the probability of containment is derived and its properties explored. In Section 4 we demonstrate that a stochastic ordering between two possible within-household severities confers an ordering on the respective containment probabilities. In Section 5 numerical examples are presented which demonstrate the theory, including an example concerned with pandemic influenza. The paper concludes with some discussion.

\section{Branching process model}

In this section we define the branching process which is used to describe the epidemic model. For the purposes of calculating a containment probability, it turns out to be sufficient to define the branching process at the level of household units in the epidemic. However, to motivate our construction, we start with an informal description of the underlying epidemic model.

\subsection{Underlying epidemic model}

Consider a population consisting of $N$ individuals of $I$ types (such as adults/children, for instance), partitioned into households. The households can be of different sizes. At any time $t \geq 0$, each individual in the population is either susceptible, exposed, infective, or removed. Susceptible individuals can contract the disease, exposed individuals have been infected but cannot infect others, infectives can infect others, and removed individuals are no longer infectious, and play no further part in the epidemic. At this stage we shall not explicitly specify exposed or infectious periods (i.e. the random time an individual spends being exposed or infective, respectively), since they will not ultimately be required.

We suppose that each individual in the population can have potentially infectious contacts of three kinds. These are internal contacts, which are with any other member of the population, within-household contacts, and external contacts, the latter being with individuals outside the population itself. We assume that any two individuals have internal contacts at times given by the points of a Poisson process with a rate of the form $\lambda / N$, where $\lambda$ can depend on the types of individual in question. Each such contact between a susceptible and an infective individual results in the former immediately becoming exposed. Under the assumption that 
all such contact processes are independent, as $N \rightarrow \infty$ we can approximate the epidemic model by a multitype branching process in which individuals correspond to households in the underlying epidemic model. As described in full detail in [3], the basic idea is that, for large $N$, the probability that a single household receives more than one internal contact converges to 0 , and so each infected household then gives rise to independent 'offspring' consisting of other household epidemics. Specifically, the types of individual in the branching process correspond to different initial configurations of household epidemics, i.e. the numbers of individuals of each type in the household and the identity of the single initial infective.

For our purposes, it is not necessary to specify a model for the transmission within a household. Instead, we assume that the final outcome of each within-household epidemic can be summarised by a random vector, which we call the household severity. The elements of this vector can be thought of as representing the total time during which the within-household epidemic exerts pressure on susceptible individuals in the population. We assume that there are $K \leq I$ different types of severity, so that the severity vector is $K$-dimensional. One possible interpretation of this assumption is that each element of the severity vector represents the total time spent by infective individuals in the household in a certain location (e.g. school, workplace, etc.).

Finally, we assume that, independently of other individuals, each individual in the population can make external contacts at times given by the points of a homogeneous Poisson process. The relevant details, in terms of the branching process, are given below.

\subsection{Branching process}

We now give precise details of the branching process, relating these to the epidemic described above. Recall that individuals in the branching process correspond to households in the epidemic. Let $H$ denote the number of types in the branching process, corresponding to initial configurations of within-household epidemics.

For $1 \leq h \leq H$ and $1 \leq k \leq K$, let the random variable $S_{h, k}$ denote the amount of type- $k$ severity produced by a type- $h$ individual in the branching process, and let $S_{h}=$ $\left(S_{h, 1}, \ldots, S_{h, K}\right)$. For $1 \leq k \leq K$ and $1 \leq u \leq H$, let $\beta_{k, u}$ denote the rate at which one unit of type- $k$ severity gives rise to type- $u$ offspring $(1 \leq u \leq H)$, and let $\boldsymbol{\beta}_{u}=\left(\beta_{1, u}, \ldots, \beta_{K, u}\right)$. Let $X_{h, u}$ denote the number of type- $u$ individuals produced by a type- $h$ individual, so that $X_{h, u}$ has a Poisson distribution with mean $\boldsymbol{\beta}_{u} \cdot \boldsymbol{S}_{h}$, where, for $K$-vectors $\boldsymbol{x}$ and $\boldsymbol{y}, \boldsymbol{x} \cdot \boldsymbol{y}=\sum_{k=1}^{K} x_{k} y_{k}$.

For $1 \leq k \leq K$, let $\alpha_{k}$ denote the rate at which one unit of type- $k$ severity creates infectious external contacts, and let $\boldsymbol{\alpha}=\left(\alpha_{1}, \ldots, \alpha_{K}\right)$. Thus, the total number of external contacts made by a type- $h$ individual has a Poisson distribution with mean $\boldsymbol{\alpha} \cdot \boldsymbol{S}_{h}$.

We require the following notation. For $\boldsymbol{\theta}=\left(\theta_{1}, \ldots, \theta_{K}\right) \in[0, \infty)^{K}$, define

$$
\psi_{h}(\boldsymbol{\theta})=\mathrm{E}\left[\exp \left\{-\boldsymbol{\theta} \cdot \boldsymbol{S}_{h}\right\}\right]
$$

For $1 \leq h \leq H$ and $1 \leq k \leq K$, define $\Delta_{h, k}$ to be the total amount of type- $k$ severity produced throughout the entire course of a branching process initiated by a single individual of type $h$, and write $\boldsymbol{\Delta}_{h}=\left(\Delta_{h, 1}, \ldots, \Delta_{h, K}\right)$. Note that, since it is possible that the branching process becomes infinite, there may be a positive probability that, for some $k, \Delta_{h, k}=\infty$. If in such circumstances $\alpha_{k}=0$, we define $\boldsymbol{\alpha} \cdot \boldsymbol{\Delta}_{h}=\infty$. Intuitively, this means that if the number of infected individuals in the population becomes extremely large then it is impossible for the disease to be contained, even if the external contact rate for type- $k$ severity is assumed to be negligible. For a more detailed discussion, see Section 3.3. 


\subsection{Reproduction number}

For the multitype branching process defined above, denote by $\boldsymbol{M}$ the mean offspring matrix with elements $m_{i j}$ denoting the expected number of type- $j$ offspring of a type- $i$ individual. By standard theory, the largest eigenvalue of $\boldsymbol{M}, R_{*}$ say, is a threshold parameter for the branching process in the sense that the process goes extinct almost surely if and only if $R_{*} \leq 1$. This parameter can also be viewed as a threshold parameter for the underlying epidemic model, as described in [2] and [3].

Here we have $m_{i j}=\mathrm{E}\left[X_{i j}\right]=\sum_{k=1}^{K} \mathrm{E}\left[S_{i, k}\right] \beta_{k, j}$. Let $S$ denote the $H \times K$ matrix with $(h, k)$ th entry $\mathrm{E}\left[S_{h, k}\right]$, and let $\boldsymbol{B}$ denote the $K \times H$ matrix with $(k, h)$ th entry $\beta_{k, h}$. Thus, $\boldsymbol{M}=\boldsymbol{S B}$, and $R_{*}$ is the largest eigenvalue of this matrix. Alternatively, $R_{*}$ can be viewed as the largest eigenvalue of the expected severity matrix $\boldsymbol{M}^{*}$, where $m_{i j}^{*}$ is the expected amount of type- $j$ severity produced from one unit of type- $i$ severity. Thus, $\boldsymbol{M}^{*}=\boldsymbol{B} S$. By Theorem A.6.2 of [8], the nonzero eigenvalues of $\boldsymbol{M}$ and $\boldsymbol{M}^{*}$ are the same and, therefore, $R_{*}$ will be the same for either definition.

\section{Probability of containment}

The total number of external contacts produced throughout the entire course of the branching process has a Poisson distribution with mean $\boldsymbol{\alpha} \cdot \boldsymbol{\Delta}_{h}$, given that the process starts with a single individual of type $h$. Thus, conditional on $\boldsymbol{\Delta}_{h}$, the probability of containment, i.e. the probability that there are no external contacts, is $\exp \left(-\boldsymbol{\alpha} \cdot \boldsymbol{\Delta}_{h}\right)$. Thus,

$$
\mathrm{P}(\text { containment } \mid \text { initial individual is type } h)=\mathrm{E}\left[\exp \left(-\boldsymbol{\alpha} \cdot \boldsymbol{\Delta}_{h}\right)\right] \text {. }
$$

For $h=1, \ldots, H$ and $\boldsymbol{\theta} \in[0, \infty)^{K}$, define $G_{h}(\boldsymbol{\theta})=\mathrm{E}\left[\exp \left(-\boldsymbol{\theta} \cdot \boldsymbol{\Delta}_{h}\right)\right]$, so that the containment probability is given by $G_{h}(\boldsymbol{\alpha})$.

Theorem 1. For $h=1, \ldots, H$ and $\boldsymbol{\theta} \in[0, \infty)^{K}$, the generating functions $G_{h}(\boldsymbol{\theta})$ satisfy

$$
G_{h}(\boldsymbol{\theta})=\psi_{h}\left(\boldsymbol{\theta}+\sum_{u=1}^{H}\left(1-G_{u}(\boldsymbol{\theta})\right) \boldsymbol{\beta}_{u}\right) .
$$

Proof. Recall that $X_{h, u}$ is the number of type- $u$ offspring of a type- $h$ individual. Since the offspring of an individual behave independently, the branching process can be split into the initial type- $h$ household plus $\sum_{u=1}^{H} X_{h, u}$ new branching processes initiated by the offspring of the initial individual. Thus, the total severity satisfies

$$
\boldsymbol{\Delta}_{h}=\boldsymbol{S}_{h}+\sum_{u=1}^{H} \sum_{j=1}^{X_{h, u}} \boldsymbol{\Delta}_{u}^{(j)},
$$

where the $\boldsymbol{\Delta}_{u}^{(j)}$ are independent and identically distributed copies of $\boldsymbol{\Delta}_{u}$, and the empty sum is 0 . Note that the $\boldsymbol{\Delta}_{u}^{(j)}$ are also independent of the $X_{h, u}$. Thus,

$$
\begin{aligned}
G_{h}(\boldsymbol{\theta}) & =\mathrm{E}\left[\exp \left(-\boldsymbol{\theta} \cdot \boldsymbol{\Delta}_{h}\right)\right] \\
& =\mathrm{E}\left[\exp \left(-\boldsymbol{\theta} \cdot\left(\boldsymbol{S}_{h}+\sum_{u=1}^{H} \sum_{j=1}^{X_{h, u}} \boldsymbol{\Delta}_{u}^{(j)}\right)\right)\right] \\
& =\mathrm{E}\left[\exp \left(-\boldsymbol{\theta} \cdot \boldsymbol{S}_{h}\right) \prod_{u=1}^{H} \prod_{j=1}^{X_{h, u}} \exp \left(-\boldsymbol{\theta} \cdot \boldsymbol{\Delta}_{u}^{(j)}\right)\right]
\end{aligned}
$$




$$
\begin{aligned}
& =\mathrm{E}\left[\exp \left(-\boldsymbol{\theta} \cdot \boldsymbol{S}_{h}\right) \mathrm{E}\left[\prod_{u=1}^{H} \prod_{j=1}^{X_{h, u}} \exp \left(-\boldsymbol{\theta} \cdot \boldsymbol{\Delta}_{u}^{(j)}\right) \mid \boldsymbol{S}_{h}, X_{h, 1}, \ldots, X_{h, H}\right]\right] \\
& =\mathrm{E}\left[\exp \left(-\boldsymbol{\theta} \cdot \boldsymbol{S}_{h}\right) \prod_{u=1}^{H} \prod_{j=1}^{X_{h, u}} \mathrm{E}\left[\exp \left(-\boldsymbol{\theta} \cdot \boldsymbol{\Delta}_{u}^{(j)}\right) \mid \boldsymbol{S}_{h}, X_{h, 1}, \ldots, X_{h, H}\right]\right] \\
& =\mathrm{E}\left[\exp \left(-\boldsymbol{\theta} \cdot \boldsymbol{S}_{h}\right) \prod_{u=1}^{H} G_{u}(\boldsymbol{\theta})^{X_{h, u}}\right] \\
& =\mathrm{E}\left[\exp \left(-\boldsymbol{\theta} \cdot \boldsymbol{S}_{h}\right) \mathrm{E}\left[\prod_{u=1}^{H} G_{u}(\boldsymbol{\theta})^{X_{h, u}} \mid \boldsymbol{S}_{h}\right]\right] \\
& =\mathrm{E}\left[\exp \left(-\boldsymbol{\theta} \cdot \boldsymbol{S}_{h}\right) \prod_{u=1}^{H} \exp \left(-\left(1-G_{u}(\boldsymbol{\theta})\right) \boldsymbol{\beta}_{u} \cdot \boldsymbol{S}_{h}\right)\right]
\end{aligned}
$$

since, conditional on $S_{h}$, the $X_{h, u}(u=1, \ldots, H)$ have independent Poisson distributions with means $\boldsymbol{\beta}_{u} \cdot \boldsymbol{S}_{h}$ and probability generating functions $\mathrm{E}\left[s^{X_{h, u}}\right]=\exp \left((1-s) \boldsymbol{\beta}_{u} \cdot \boldsymbol{S}_{h}\right)$. Therefore,

$$
G_{h}(\boldsymbol{\theta})=\mathrm{E}\left[\exp \left(-\left(\boldsymbol{\theta}+\sum_{u=1}^{H}\left(1-G_{u}(\boldsymbol{\theta})\right) \boldsymbol{\beta}_{u}\right) \cdot \boldsymbol{S}_{h}\right)\right]=\psi_{h}\left(\boldsymbol{\theta}+\sum_{u=1}^{H}\left(1-G_{u}(\boldsymbol{\theta})\right) \boldsymbol{\beta}_{u}\right) .
$$

\subsection{Uniqueness}

For a given arbitrary value of $\boldsymbol{\theta}$, equations (1) do not uniquely determine $\boldsymbol{G}(\boldsymbol{\theta})=\left(G_{1}(\boldsymbol{\theta})\right.$, $\left.\ldots, G_{H}(\boldsymbol{\theta})\right)$. However, in this section we show that in most practical situations equations (1) have a unique solution. For convenience, we rewrite equations (1) as the vector equation

$$
\boldsymbol{G}(\boldsymbol{\theta})=\boldsymbol{f}(\boldsymbol{\theta}, \boldsymbol{G}(\boldsymbol{\theta}))
$$

where, for $\boldsymbol{x}=\left(x_{1}, \ldots, x_{H}\right) \in[0,1]^{H}, \boldsymbol{f}(\boldsymbol{\theta}, \boldsymbol{x})=\left(f_{1}(\boldsymbol{\theta}, \boldsymbol{x}), \ldots, f_{H}(\boldsymbol{\theta}, \boldsymbol{x})\right)$ and

$$
f_{h}(\boldsymbol{\theta}, \boldsymbol{x})=\psi_{h}\left(\boldsymbol{\theta}+\sum_{u=1}^{H}\left(1-x_{u}\right) \boldsymbol{\beta}_{u}\right) .
$$

Recall that a branching process is irreducible if, for every pair of types $(u, v)$, there exists a $t \in \mathbb{N}$ such that the probability that there is at least one type- $v$ individual in generation $t$ is strictly positive, when the branching process has one initial ancestor of type $u$.

Theorem 2. The equation $\boldsymbol{x}=\boldsymbol{f}(\boldsymbol{\theta}, \boldsymbol{x})$ has a unique solution for $\boldsymbol{x}$ in $[0,1]^{H}$ if any of the following conditions hold:

(i) $R_{*} \leq 1$;

(ii) $\boldsymbol{\theta} \in(0, \infty)^{K}$;

(iii) the branching process is irreducible with $\boldsymbol{\theta} \neq \mathbf{0}$.

Two lemmas are needed in order to prove this theorem, but first we define some more notation. For $h, u \in\{1, \ldots, H\}$ and $t=0,1, \ldots$, let $Z_{h, u}(t)$ be the number of type- $u$ individuals in generation $t$ of the branching process with generation 0 consisting of a single type- $h$ individual. 
For $k \in\{1, \ldots, K\}$ and $t \in \mathbb{N}$, let the cumulative severity $\Delta_{h, k}(t)$ be the sum of the type- $k$ severity generated by the first $t-1$ generations of the branching process described above, and adopt the convention that $\Delta_{h, k}(0)=0$. Form the vectors $Z_{h}(t)=\left(Z_{h, 1}(t), \ldots, Z_{h, H}(t)\right)$ and $\boldsymbol{\Delta}_{h}(t)=\left(\Delta_{h, 1}(t), \ldots, \Delta_{h, K}(t)\right)$. We adopt the following definitions for $H$-vectors $\boldsymbol{x}$ and $\boldsymbol{y}$ :

$$
\boldsymbol{x}^{\boldsymbol{y}}=\prod_{h=1}^{H} x_{h}^{y_{h}}, \quad \boldsymbol{x} \leq \boldsymbol{y} \text { if and only if } x_{h} \leq y_{h} \text { for all } h=1, \ldots, H .
$$

For $t=0,1, \ldots$ and $\boldsymbol{x} \in[0,1]^{H}$, let $f_{h}^{t}(\boldsymbol{\theta}, \boldsymbol{x})=\mathrm{E}\left[\exp \left(-\boldsymbol{\theta} \cdot \boldsymbol{\Delta}_{h}(t)\right) \boldsymbol{x}^{\boldsymbol{Z}_{h}(t)}\right]$ and let $\boldsymbol{f}^{t}(\boldsymbol{\theta}, \boldsymbol{x})=$ $\left(f_{1}^{t}(\boldsymbol{\theta}, \boldsymbol{x}), \ldots, f_{H}^{t}(\boldsymbol{\theta}, \boldsymbol{x})\right)$.

Lemma 1. For $t \in \mathbb{N}, \boldsymbol{\theta} \in[0, \infty)^{K}$, and $\boldsymbol{x} \in[0,1]^{H}$,

$$
\boldsymbol{f}^{t}(\boldsymbol{\theta}, \boldsymbol{x})=\boldsymbol{f}\left(\boldsymbol{\theta}, \boldsymbol{f}^{t-1}(\boldsymbol{\theta}, \boldsymbol{x})\right) .
$$

Proof. For $h \in\{1, \ldots, H\}$,

$$
\begin{aligned}
f_{h}^{t}(\boldsymbol{\theta}, \boldsymbol{x}) & =\mathrm{E}\left[\exp \left(-\boldsymbol{\theta} \cdot \boldsymbol{\Delta}_{h}(t)\right) \boldsymbol{x}^{\boldsymbol{Z}_{h}(t)}\right] \\
& =\mathrm{E}\left[\exp \left(\boldsymbol{\theta} \cdot\left(\boldsymbol{S}_{h}+\sum_{u=1}^{H} \sum_{j=1}^{X_{h, u}} \boldsymbol{\Delta}_{u}^{(j)}(t-1)\right)\right) \boldsymbol{x}^{\sum_{u=1}^{H} \sum_{j=1}^{X_{h, u}} \boldsymbol{Z}_{u}^{(j)}(t-1)}\right],
\end{aligned}
$$

where $X_{h, u}$ is the number of type- $u$ offspring from the type- $h$ ancestor and, for $j \in \mathbb{N}$, $\boldsymbol{\Delta}_{u}^{(j)}(t-1)$ and $\boldsymbol{Z}_{u}^{(j)}(t-1)$ are independent and identically distributed copies of $\boldsymbol{\Delta}_{u}(t-1)$ and $Z_{u}(t-1)$, respectively. Arguing similarly to the proof of Theorem 1 ,

$$
\begin{aligned}
& f_{h}^{t}(\boldsymbol{\theta}, \boldsymbol{x})= \mathrm{E}\left[\exp \left(-\boldsymbol{\theta} \cdot \boldsymbol{S}_{h}\right) \prod_{u=1}^{H} \exp \left(-\sum_{j=1}^{X_{h, u}} \boldsymbol{\theta} \cdot \boldsymbol{\Delta}_{u}^{(j)}(t-1)\right) \boldsymbol{x}^{\sum_{j=1}^{X_{h, u}} \boldsymbol{Z}_{u}^{(j)}(t-1)}\right] \\
&=\mathrm{E} {\left[\exp \left(-\boldsymbol{\theta} \cdot \boldsymbol{S}_{h}\right)\right.} \\
&\left.\times \mathrm{E}\left[\prod_{u=1}^{H} \exp \left(-\sum_{j=1}^{X_{h, u}} \boldsymbol{\theta} \cdot \boldsymbol{\Delta}_{u}^{(j)}(t-1)\right) \boldsymbol{x}^{\sum_{j=1}^{X_{h, u}} \boldsymbol{Z}_{u}^{(j)}(t-1)} \mid \boldsymbol{S}_{h}, X_{h, 1}, \ldots, X_{h, H}\right]\right] \\
&=\mathrm{E}\left[\exp \left(-\boldsymbol{\theta} \cdot \boldsymbol{S}_{h}\right)\right.\left.\times \prod_{u=1}^{H} \prod_{j=1}^{X_{h, u}} \mathrm{E}\left[\exp \left(-\boldsymbol{\theta} \cdot \boldsymbol{\Delta}_{u}^{(j)}(t-1)\right) \boldsymbol{x}^{\boldsymbol{Z}_{u}^{(j)}(t-1)} \mid \boldsymbol{S}_{h}, X_{h, 1}, \ldots, X_{h, H}\right]\right] \\
&=\mathrm{E}\left[\operatorname { e x p } ( - \boldsymbol { \theta } \cdot \boldsymbol { S } _ { h } ) \mathrm { E } \left[\prod_{u=1}^{H} f_{u}^{t-1}(\boldsymbol{\theta}, \boldsymbol{x})^{\left.\left.X_{h, u} \mid \boldsymbol{S}_{h}\right]\right]}\right.\right. \\
&=\mathrm{E}\left[\exp \left(-\boldsymbol{\theta} \cdot \boldsymbol{S}_{h}\right) \prod_{u=1}^{H} \exp \left(-\left(1-f_{u}^{t-1}(\boldsymbol{\theta}, \boldsymbol{x})\right) \boldsymbol{\beta}_{u} \cdot \boldsymbol{S}_{h}\right)\right]
\end{aligned}
$$

since, given $\boldsymbol{S}_{h}, X_{h, u}$ has a Poisson distribution with mean $\boldsymbol{\beta}_{u} \cdot \boldsymbol{S}_{h}$ for $u=1, \ldots, H$, and these 
Poisson random variables are conditionally independent. Hence,

$$
\begin{aligned}
f_{h}^{t}(\boldsymbol{\theta}, \boldsymbol{x}) & =\mathrm{E}\left[\exp \left(-\left(\boldsymbol{\theta}+\sum_{u=1}^{H}\left(1-f_{u}^{t-1}(\boldsymbol{\theta}, \boldsymbol{x})\right) \boldsymbol{\beta}_{u}\right) \cdot \boldsymbol{S}_{h}\right)\right] \\
& =\psi_{h}\left(\boldsymbol{\theta}+\sum_{u=1}^{H}\left(1-f_{u}^{t-1}(\boldsymbol{\theta}, \boldsymbol{x})\right) \boldsymbol{\beta}_{u}\right) \\
& =f_{h}\left(\boldsymbol{\theta}, \boldsymbol{f}^{t-1}(\boldsymbol{\theta}, \boldsymbol{x})\right),
\end{aligned}
$$

as required.

Lemma 2. Let $E_{h}$ be the event that the branching process with one initial ancestor of type $h$ becomes extinct, and let $F_{h}=\left\{\Delta_{h, k}<\infty\right.$ for $\left.k=1, \ldots, K\right\}$. Then $\mathrm{P}\left(F_{h} \mid E_{h}^{\mathrm{c}}\right)=0$.

Proof. If $E_{h}^{\mathrm{c}}$ occurs, there must exist a type of individual, $u$ say, such that there exists a line of descent which contains infinitely many individuals of type $u$. For $t \in \mathbb{N}$, let $\boldsymbol{Y}_{u}(t)=\left(Y_{u, 1}(t), \ldots, Y_{u, K}(t)\right)$ be the severity generated by the $t$ th such type- $u$ individual, i.e. conditional upon its being in the line of descent. Since offspring behave independently of their parent, it follows that the $\boldsymbol{Y}_{u}(t)$ are independent and identically distributed, and moreover, $\mathrm{P}\left(\boldsymbol{Y}_{u}(t)=\mathbf{0} \mid E_{h}^{\mathrm{c}}\right)=0$. Therefore, for at least one component (say $k^{*}$ ), we have $\sum_{t=1}^{\infty} Y_{u, k^{*}}(t)=\infty$, P-almost surely on $E_{h}^{\mathrm{c}}$. However, on $E_{h}^{\mathrm{c}}, \Delta_{h, k^{*}} \geq \sum_{t=1}^{\infty} Y_{u^{*}, k^{*}}(t)=\infty$, P-almost surely, and so $\mathrm{P}\left(F_{h} \mid E_{h}^{\mathrm{c}}\right)=0$, as required.

Proof of Theorem 2. This proof is similar to the proof of Theorem 7.2 of [9].

We begin by showing that, irrespective of the value of $\boldsymbol{x} \in[0,1]^{H}, \lim _{t \rightarrow \infty} f_{h}^{t}(\boldsymbol{\theta}, \boldsymbol{x})=$ $G_{h}(\boldsymbol{\theta})$, where $f_{h}^{t}(\boldsymbol{\theta}, \boldsymbol{x})$ is as defined in Lemma 1. First assume that $R_{*} \leq 1$, in which case extinction is certain; see, for example, Theorem 7.1 of [9]. Thus,

$$
\lim _{t \rightarrow \infty} f_{h}^{t}(\boldsymbol{\theta}, \boldsymbol{x})=\lim _{t \rightarrow \infty} \mathrm{E}\left[\exp \left(-\boldsymbol{\theta} \cdot \boldsymbol{\Delta}_{h}(t)\right) \boldsymbol{x}^{\boldsymbol{Z}_{h}(t)}\right]=\mathrm{E}\left[\lim _{t \rightarrow \infty} \exp \left(-\boldsymbol{\theta} \cdot \boldsymbol{\Delta}_{h}(t)\right) \boldsymbol{x}^{\boldsymbol{Z}_{h}(t)}\right],
$$

by the dominated convergence theorem. Next note that, since extinction is certain, we have $\lim _{t \rightarrow \infty} \boldsymbol{Z}_{h}(t)=\mathbf{0}$ almost surely and so $\lim _{t \rightarrow \infty} f_{h}^{t}(\boldsymbol{\theta}, \boldsymbol{x})=\mathrm{E}\left[\exp \left(-\boldsymbol{\theta} \cdot \boldsymbol{\Delta}_{h}\right)\right]=G_{h}(\boldsymbol{\theta})$.

We can extend this argument to the case where $R_{*}$ is arbitrary and $\boldsymbol{\theta} \in(0, \infty)^{K}$; or the case where $R_{*}$ is arbitrary, $\boldsymbol{\theta} \neq \mathbf{0}$, and the branching process is irreducible. By Lemma 2 , conditioning on nonextinction implies that at least one component of the severity vector is almost surely infinite. Therefore, if $\boldsymbol{\theta}>\boldsymbol{0}$ then

$$
\mathrm{E}\left[\lim _{t \rightarrow \infty} \exp \left(-\boldsymbol{\theta} \cdot \boldsymbol{\Delta}_{h}(t)\right) \mid E_{h}^{\mathrm{c}}\right]=0 \quad \text { and } \quad \mathrm{E}\left[\exp \left(-\boldsymbol{\theta} \cdot \boldsymbol{\Delta}_{h}\right) \mid E_{h}^{\mathrm{c}}\right]=0 .
$$

Similarly, if the branching process is irreducible then conditioning on nonextinction implies there must be infinitely many of every type of individual and so, by a renewal argument, every component of the total severity vector must be infinite almost surely. Therefore, when the process is irreducible and $\boldsymbol{\theta} \neq \mathbf{0}$, (3) again holds. Finally, note that, for $\boldsymbol{x} \in[0,1]^{H}$, (3) implies that

$$
0 \leq \mathrm{E}\left[\lim _{t \rightarrow \infty} \exp \left(-\boldsymbol{\theta} \cdot \boldsymbol{\Delta}_{h}(t)\right) \boldsymbol{x}^{\boldsymbol{Z}_{h}(t)} \mid E_{h}^{\mathrm{c}}\right] \leq \mathrm{E}\left[\lim _{t \rightarrow \infty} \exp \left(-\boldsymbol{\theta} \cdot \boldsymbol{\Delta}_{h}(t)\right) \mid E_{h}^{\mathrm{c}}\right]=0,
$$

i.e.

$$
\mathrm{E}\left[\lim _{t \rightarrow \infty} \exp \left(-\boldsymbol{\theta} \cdot \boldsymbol{\Delta}_{h}(t)\right) \boldsymbol{x}^{\boldsymbol{Z}_{h}(t)} \mid E_{h}^{\mathrm{c}}\right]=0
$$


By applying the dominated convergence theorem, conditioning on $E_{h}$ and using (4) and then (3), we find that, for any $\boldsymbol{x} \in[0,1]^{H}$,

$$
\begin{aligned}
& \lim _{t \rightarrow \infty} f_{h}^{t}(\boldsymbol{\theta}, \boldsymbol{x})=\mathrm{E}\left[\lim _{t \rightarrow \infty} \exp \left(-\boldsymbol{\theta} \cdot \boldsymbol{\Delta}_{h}(t)\right) \boldsymbol{x}^{\boldsymbol{Z}_{h}(t)} \mid E_{h}\right] \mathrm{P}\left(E_{h}\right) \\
& +\mathrm{E}\left[\lim _{t \rightarrow \infty} \exp \left(-\boldsymbol{\theta} \cdot \boldsymbol{\Delta}_{h}(t)\right) \boldsymbol{x}^{\boldsymbol{Z}_{h}(t)} \mid E_{h}^{\mathrm{c}}\right] \mathrm{P}\left(E_{h}^{\mathrm{c}}\right) \\
& =\mathrm{E}\left[\exp \left(-\boldsymbol{\theta} \cdot \boldsymbol{\Delta}_{h}\right) \mid E_{h}\right] \mathrm{P}\left(E_{h}\right)+0 \cdot \mathrm{P}\left(E_{h}^{\mathrm{c}}\right) \\
& =\mathrm{E}\left[\exp \left(-\boldsymbol{\theta} \cdot \boldsymbol{\Delta}_{h}\right) \mid E_{h}\right] \mathrm{P}\left(E_{h}\right)+\mathrm{E}\left[\exp \left(-\boldsymbol{\theta} \cdot \boldsymbol{\Delta}_{h}\right) \mid E_{h}^{\mathrm{c}}\right] \mathrm{P}\left(E_{h}^{\mathrm{c}}\right) \\
& =\mathrm{E}\left[\exp \left(-\boldsymbol{\theta} \cdot \boldsymbol{\Delta}_{h}\right)\right] \\
& =G_{h}(\boldsymbol{\theta}) \text {. }
\end{aligned}
$$

Therefore, we have shown that, for a branching process with $R_{*} \leq 1$, or with $R_{*}$ arbitrary and $\boldsymbol{\theta} \in(0, \infty)^{K}$, or with $R_{*}$ arbitrary, irreducibility, and $\boldsymbol{\theta} \neq \mathbf{0}$,

$$
\lim _{t \rightarrow \infty} f^{t}(\boldsymbol{\theta}, \boldsymbol{x})=\boldsymbol{G}(\boldsymbol{\theta})
$$

for all $\boldsymbol{x} \in[0,1]^{H}$.

Theorem 1 implies that there is at least one solution to $\boldsymbol{f}(\boldsymbol{\theta}, \boldsymbol{x})=\boldsymbol{x}$, and so assume that $\boldsymbol{G}^{*}(\boldsymbol{\theta})$ is another solution. We will show by induction on $t$ that $\boldsymbol{f}^{t}\left(\boldsymbol{\theta}, \boldsymbol{G}^{*}(\boldsymbol{\theta})\right)=\boldsymbol{G}^{*}(\boldsymbol{\theta})$. For the initialisation, note that $f_{h}^{0}\left(\boldsymbol{\theta}, \boldsymbol{G}^{*}(\boldsymbol{\theta})\right)=\mathrm{E}\left[\exp \left(-\boldsymbol{\theta} \cdot \boldsymbol{\Delta}_{h}(0)\right) \boldsymbol{G}^{*}(\boldsymbol{\theta})^{\boldsymbol{Z}_{h}(0)}\right]=G_{h}^{*}(\boldsymbol{\theta})$, and so $f^{0}\left(\boldsymbol{\theta}, G^{*}(\boldsymbol{\theta})\right)=\boldsymbol{G}^{*}(\boldsymbol{\theta})$. Assume that the inductive hypothesis holds and that, for $t \in \mathbb{N}$, $\boldsymbol{f}^{t-1}\left(\boldsymbol{\theta}, \boldsymbol{G}^{*}(\boldsymbol{\theta})\right)=\boldsymbol{G}^{*}(\boldsymbol{\theta})$, and note that, by Lemma 1 ,

$$
\boldsymbol{f}^{t}\left(\boldsymbol{\theta}, \boldsymbol{G}^{*}(\boldsymbol{\theta})\right)=\boldsymbol{f}\left(\boldsymbol{\theta}, \boldsymbol{f}^{t-1}\left(\boldsymbol{\theta}, \boldsymbol{G}^{*}(\boldsymbol{\theta})\right)\right)=\boldsymbol{f}\left(\boldsymbol{\theta}, \boldsymbol{G}^{*}(\boldsymbol{\theta})\right)=\boldsymbol{G}^{*}(\boldsymbol{\theta}),
$$

and so, by induction, $\boldsymbol{f}^{t}\left(\boldsymbol{\theta}, \boldsymbol{G}^{*}(\boldsymbol{\theta})\right)=\boldsymbol{G}^{*}(\boldsymbol{\theta})$ for $t=0,1, \ldots$ However, from (5),

$$
\boldsymbol{G}^{*}(\boldsymbol{\theta})=\lim _{t \rightarrow \infty} \boldsymbol{f}^{t}\left(\boldsymbol{\theta}, \boldsymbol{G}^{*}(\boldsymbol{\theta})\right)=\boldsymbol{G}(\boldsymbol{\theta}) .
$$

This completes the proof of Theorem 2.

\subsection{Probability of extinction}

In this section we show how the containment probability relates to the probability of extinction.

Theorem 3. The probability of extinction for a branching process with one initial ancestor of type $h$ corresponds to $\lim _{\boldsymbol{\theta} \rightarrow \mathbf{0}} G_{h}(\boldsymbol{\theta})$, where $\lim _{\boldsymbol{\theta} \rightarrow \mathbf{0}}$ represents the limit as $\boldsymbol{\theta} \rightarrow \mathbf{0}$ with $\boldsymbol{\theta} \in(0, \infty)^{K}$.

Proof. To show that $\lim _{\boldsymbol{\theta} \rightarrow \mathbf{0}} G_{h}(\boldsymbol{\theta})$ gives the probability of extinction for the branching process, note that, by Lemma 2, conditioning on $E_{h}^{\mathrm{c}}$ implies that at least one component of $\boldsymbol{\Delta}_{h}$ is infinite. Therefore, for $\boldsymbol{\theta}$ with all components strictly positive,

$$
\begin{aligned}
G_{h}(\boldsymbol{\theta}) & =\mathrm{E}\left[\exp \left(-\boldsymbol{\theta} \cdot \boldsymbol{\Delta}_{h}\right) \mid E_{h}\right] \mathrm{P}\left(E_{h}\right)+\mathrm{E}\left[\exp \left(-\boldsymbol{\theta} \cdot \boldsymbol{\Delta}_{h}\right) \mid E_{h}^{\mathrm{c}}\right] \mathrm{P}\left(E_{h}^{\mathrm{c}}\right) \\
& =\mathrm{E}\left[\exp \left(-\boldsymbol{\theta} \cdot \boldsymbol{\Delta}_{h}\right) \mid E_{h}\right] \mathrm{P}\left(E_{h}\right) .
\end{aligned}
$$

Thus, by the dominated convergence theorem, $\lim _{\boldsymbol{\theta} \rightarrow \mathbf{0}} G_{h}(\boldsymbol{\theta})=\mathrm{P}\left(E_{h}\right)$. 


\subsection{Nonuniqueness}

In this section we describe and discuss the solutions to (2) when there are multiple solutions. Theorem 2 implies that if this occurs then at least one component of $\boldsymbol{\theta}$ must be 0 .

Theorem 4. There is at most one solution to each equation $x_{h}=f_{h}(\boldsymbol{\theta}, \boldsymbol{x})$ with $x_{h} \in[0,1)$ for $h=1, \ldots, H$. In particular, there is at most one solution to $(2)$ in $[0,1)^{H}$.

Proof. We begin by using a convexity argument to show the first part of the theorem.

Let

$$
\begin{gathered}
\psi_{h}^{(k)}(\tilde{\boldsymbol{\theta}})=\left.\frac{\partial \psi_{h}(\boldsymbol{\theta})}{\partial \theta_{k}}\right|_{\boldsymbol{\theta}=\tilde{\boldsymbol{\theta}}}=-\mathrm{E}\left[S_{h, k} \exp \left(-\tilde{\boldsymbol{\theta}} \cdot \boldsymbol{S}_{h}\right)\right] \leq 0, \\
\psi_{h}^{(k, l)}(\tilde{\boldsymbol{\theta}})=\left.\frac{\partial^{2} \psi_{h}(\boldsymbol{\theta})}{\partial \theta_{k} \partial \theta_{l}}\right|_{\boldsymbol{\theta}=\tilde{\boldsymbol{\theta}}}=\mathrm{E}\left[S_{h, k} S_{h, l} \exp \left(-\tilde{\boldsymbol{\theta}} \cdot \boldsymbol{S}_{h}\right)\right] \geq 0 .
\end{gathered}
$$

Then

$$
\begin{gathered}
\frac{\partial f_{h}(\boldsymbol{\theta}, \boldsymbol{x})}{\partial x_{i}}=-\sum_{k=1}^{K} \beta_{k, i} \psi_{h}^{(k)}\left(\boldsymbol{\theta}+\sum_{u=1}^{H}\left(1-x_{u}\right) \boldsymbol{\beta}_{u}\right) \geq 0 \\
\frac{\partial^{2} f_{h}(\boldsymbol{\theta}, \boldsymbol{x})}{\partial x_{i} \partial x_{j}}=\sum_{k=1}^{K} \sum_{l=1}^{K} \beta_{k, i} \beta_{l, j} \psi_{h}^{(k, l)}\left(\boldsymbol{\theta}+\sum_{u=1}^{H}\left(1-x_{u}\right) \boldsymbol{\beta}_{u}\right) \geq 0
\end{gathered}
$$

hence, $\boldsymbol{f}_{h}(\boldsymbol{\theta}, \boldsymbol{x})$ is nondecreasing and convex in $x_{h}$. In addition, we have $0 \leq f_{h}(\boldsymbol{\theta}, \boldsymbol{x}) \leq 1$, and so $x_{h}=f_{h}(\boldsymbol{\theta}, \boldsymbol{x})$ can have precisely one, two, or infinitely many solutions for $x_{h} \in[0,1]$, and either zero, one, or infinitely many for $x_{h} \in[0,1)$. However, we can rule out the case with infinitely many solutions (where $f_{h}(\boldsymbol{\theta}, \boldsymbol{x})$ as a function of $x_{h}$ follows part of the line $y=x$ ) because if $\psi_{h}^{(k)}\left(\boldsymbol{\theta}+\sum_{u=1}^{H}\left(1-x_{u}\right) \boldsymbol{\beta}_{u}\right)>0$ for some $k$ then $\psi_{h}^{(k, k)}\left(\boldsymbol{\theta}+\sum_{u=1}^{H}\left(1-x_{u}\right) \boldsymbol{\beta}_{u}\right)>0$, and so $\partial f_{h}(\boldsymbol{\theta}, \boldsymbol{x}) / \partial x_{h}>0$ implies that $\partial^{2} f_{h}(\boldsymbol{\theta}, \boldsymbol{x}) / \partial x_{h}^{2}>0$. The second part of the theorem follows easily from the first.

Theorem 4 demonstrates that there are at most two solutions to each equation $x_{h}=f_{h}(\boldsymbol{\alpha}, \boldsymbol{x})$, and if two solutions exist then one of them must be $x_{h}=1$. As each of these $H$ equations depends on $x_{1}, \ldots, x_{H}$, then a priori there will be interdependence between the number and the value of the solutions to each equation. The key question remaining to be answered is: when multiple solutions do exist, which solution do we interpret as the probability of containment?

Consider the probability of containment for a branching process starting from a single type- $h$ ancestor. If $\alpha_{k}=0$ for each type of severity which it is possible to ultimately produce from a type- $h$ ancestor, then no external contacts can occur. However, it may still be possible for infinitely many individuals to be produced in the branching process. In such circumstances the probability of containment can either be interpreted as 1 (since no external contacts can occur) or as the extinction probability of the branching process, $\mathrm{P}\left(E_{h}\right)$. These correspond to the two solutions to the equation $x_{h}=f_{h}(\boldsymbol{\alpha}, \boldsymbol{x})$. From the epidemic modelling point of view, it appears unnatural to consider the epidemic to be successfully contained when infinitely many individuals have been infected. We will therefore opt for the second of these two interpretations, in which the smallest solution to $x_{h}=f_{h}(\boldsymbol{\alpha}, \boldsymbol{x})$ is called the probability of containment.

\subsection{Calculating the containment probability numerically}

We finish this section with an algorithm for calculating the probability of containment. 
Lemma 3. Define the sequence $\left(\boldsymbol{x}_{n}\right)$ with the relations $\boldsymbol{x}_{0}=\mathbf{0}$ and, for $n \in \mathbb{N}, \boldsymbol{x}_{n}=$ $\boldsymbol{f}\left(\boldsymbol{\theta}, \boldsymbol{x}_{n-1}\right)$, where $\boldsymbol{f}(\boldsymbol{\theta}, \boldsymbol{x})=\boldsymbol{\psi}\left(\boldsymbol{\theta}+\sum_{u=1}^{H}\left(1-x_{n-1, u}\right) \boldsymbol{\beta}_{u}\right)$. Then

1. $\left(\boldsymbol{x}_{n}\right)$ is nondecreasing;

2. if $\boldsymbol{G}(\boldsymbol{\theta})$ satisfies $\boldsymbol{G}(\boldsymbol{\theta})=\boldsymbol{f}(\boldsymbol{\theta}, \boldsymbol{G}(\boldsymbol{\theta}))$ then $\boldsymbol{x}_{n} \leq \boldsymbol{G}(\boldsymbol{\theta})$ for all $n$;

3. $\lim _{n \rightarrow \infty} \boldsymbol{x}_{n}=\boldsymbol{G}^{*}(\boldsymbol{\theta})$, where $\boldsymbol{G}^{*}(\boldsymbol{\theta})$ is the smallest solution in $[0,1]^{H}$ to the equation $\boldsymbol{x}=\boldsymbol{f}(\boldsymbol{\theta}, \boldsymbol{x})$.

The first two parts of this lemma are easily proved by induction, and when combined with the monotone sequence theorem, yield the third part [11]. From the discussion in Section 3.3 we see that, when $\boldsymbol{\theta}=\boldsymbol{\alpha}$, Lemma 3 implies that the sequence $\left(\boldsymbol{x}_{n}\right)$ converges to the smallest solution of (2), which corresponds to the probability of containment.

To obtain numerical estimates for $\boldsymbol{G}(\boldsymbol{\alpha})$, sequentially calculate terms in the sequence $\left(\boldsymbol{x}_{n}\right)$ until $\boldsymbol{x}_{n+1} \approx \boldsymbol{x}_{n}$ to the desired level of accuracy. In applications, it has been noted that remarkably few iterations are required for the first eight decimal places of the sequence to stabilise, sometimes as few as two.

\section{Laplace transform orders}

This section is concerned with the relationship between the containment probability and the within-household severity distributions. In particular, it is proved that if a Laplace transform ordering exists between two within-household severity distributions then a Laplace transform ordering exists between the total severity distributions of branching processes with these within-household severity distributions. Consequently, the containment probabilities of these branching processes are also ordered.

Consider two independent branching process models, labelled 1 and 2, that differ only in their household severity distributions. For $j \in\{1,2\}$ and $h \in\{1, \ldots, H\}$, let $S_{h}^{(j)}=$ $\left(S_{h, 1}^{(j)}, \ldots, S_{h, K}^{(j)}\right)$ denote the household severities for model $j$, with Laplace transform $\psi_{h}^{(j)}(\boldsymbol{\theta})=$ $\mathrm{E}\left[\exp \left(-\boldsymbol{\theta} \cdot \boldsymbol{S}_{h}^{(j)}\right)\right]$. Likewise, define $\boldsymbol{\Delta}_{h}^{(j)}$ to be the total severity vector for model $j$, with Laplace transform $G_{h}^{(j)}=\mathrm{E}\left[\exp \left(-\boldsymbol{\theta} \cdot \boldsymbol{\Delta}_{h}^{(j)}\right)\right]$. Let $\boldsymbol{\psi}^{(j)}(\boldsymbol{\theta})=\left(\psi_{1}^{(j)}(\boldsymbol{\theta}), \ldots, \psi_{H}^{(j)}(\boldsymbol{\theta})\right)$ and $\boldsymbol{G}^{(j)}(\boldsymbol{\theta})=$ $\left(G_{1}^{(j)}(\boldsymbol{\theta}), \ldots, G_{H}^{(j)}(\boldsymbol{\theta})\right)$. For random vectors $\boldsymbol{X}$ and $\boldsymbol{Y}$ of length $K$, define $\boldsymbol{X} \geq_{\mathrm{Lt}} \boldsymbol{Y}$ if and only if $\mathrm{E}[\exp (-\boldsymbol{\theta} \cdot \boldsymbol{X})] \leq \mathrm{E}[\exp (-\boldsymbol{\theta} \cdot \boldsymbol{Y})]$ for all $\boldsymbol{\theta} \in[0, \infty)^{K}$; see, for example, [10].

Theorem 5. If $\boldsymbol{S}_{h}^{(1)} \geq_{\mathrm{Lt}} \boldsymbol{S}_{h}^{(2)}$ for all $h \in\{1, \ldots, H\}$ then

$$
\boldsymbol{\Delta}_{h}^{(1)} \geq_{\text {Lt }} \boldsymbol{\Delta}_{h}^{(2)}
$$

for all $h$, and, in particular, the probability of containment is smaller for model 1 than for model 2, i.e. $G_{h}^{(1)}(\boldsymbol{\alpha}) \leq G_{h}^{(2)}(\boldsymbol{\alpha})$ for all $h$.

Proof. First note that $S_{h}^{(1)} \geq_{\mathrm{Lt}} \boldsymbol{S}_{h}^{(2)}$ for all $h$ implies that

$$
\boldsymbol{\psi}^{(1)}(\boldsymbol{\theta}) \leq \boldsymbol{\psi}^{(2)}(\boldsymbol{\theta}) \quad \text { for all } \boldsymbol{\theta} \in[0, \infty)^{K} .
$$

From Theorem 1, for fixed $\boldsymbol{\alpha} \in[0, \infty)^{K}, \boldsymbol{G}^{(j)}(\boldsymbol{\alpha})$ satisfies $\boldsymbol{x}=\boldsymbol{f}^{(j)}(\boldsymbol{\alpha}, \boldsymbol{x})$, where

$$
\boldsymbol{f}^{(j)}(\boldsymbol{\alpha}, \boldsymbol{x})=\boldsymbol{\psi}^{(j)}\left(\boldsymbol{\alpha}+\sum_{u=1}^{H}\left(1-x_{u}\right) \boldsymbol{\beta}_{u}\right) .
$$


Therefore, from (7) we have, for all $\boldsymbol{x} \in[0,1]^{H}, \boldsymbol{f}^{(1)}(\boldsymbol{\alpha}, \boldsymbol{x}) \leq \boldsymbol{f}^{(2)}(\boldsymbol{\alpha}, \boldsymbol{x})$. In Section 3.3, we saw that the probability of containment is the smallest solution to these equations. Since $\boldsymbol{f}^{(1)}(\boldsymbol{\alpha}, \boldsymbol{x}) \leq \boldsymbol{f}^{(2)}(\boldsymbol{\alpha}, \boldsymbol{x})$ for all $\boldsymbol{x} \in[0,1]^{H}$, and $\boldsymbol{f}^{(1)}$ and $\boldsymbol{f}^{(2)}$ are continuous and nondecreasing in all components of $x$, the smallest solution to $x=f^{(1)}(\alpha, x)$ must be smaller than the smallest solution to $\boldsymbol{x}=\boldsymbol{f}^{(2)}(\boldsymbol{\alpha}, \boldsymbol{x})$. Thus, $\boldsymbol{G}^{(1)}(\boldsymbol{\alpha}) \leq \boldsymbol{G}^{(2)}(\boldsymbol{\alpha})$ for all $\boldsymbol{\alpha} \in[0, \infty)^{K}$ and so $\boldsymbol{\Delta}_{h}^{(1)} \geq_{\mathrm{Lt}} \boldsymbol{\Delta}_{h}^{(2)}$ for $h \in\{1, \ldots, H\}$.

\section{Numerical illustrations}

We now illustrate the theory with some numerical examples.

\subsection{Household severity distribution explicitly available}

Assume that the severity generated by every within-household epidemic is distributed according to some random variable $S$ and with moment generating function $\psi$. Since there is only one type of household $(H=1)$ and one type of severity $(K=1)$, there is a single internal contact rate $\beta$ and a single external contact rate $\alpha$. Equation (2) becomes $G(\alpha)=\psi(\alpha+(1-G(\alpha)) \beta)$. Figure 1 shows the containment probability for the case in which $S \equiv 1$, and, therefore, $\psi(\theta)=\mathrm{e}^{-\theta}$. Note that, when $\alpha=0$, we obtain the probability of extinction for the branching process, with a threshold at $R_{*}=\beta=1$.

\subsection{SIR within-household epidemics}

In this section the within-household epidemics are modelled by a multitype SIR (susceptibleinfective-removed) epidemic with homogeneous mixing. For this special case, the generating function of the household severity is available analytically.

Consider a population of $I$ types of individual, in which individuals are classed as susceptible, infective, or removed. For $i=1, \ldots, I$, let $n_{i}$ be the initial number of type- $i$ susceptibles and $m_{i}$ be the initial number of type- $i$ infectives. We assume that initially there are no removed individuals. Type- $i$ infectives make contact with type- $j$ susceptibles at the points of a Poisson process with rate $\gamma_{i, j}$, at which point the susceptible becomes an infective. Type- $i$ infectives

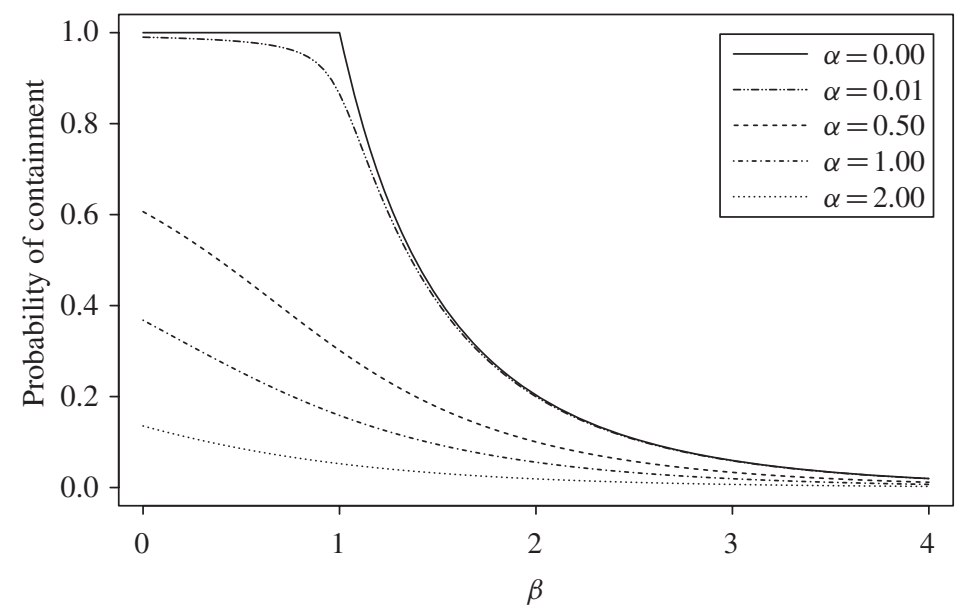

FIGURE 1: The probability of containment, $G(\alpha)$, against the internal contact rate, $\beta$, when $S \equiv 1$. 
have an infectious period of length $T_{i}$, after which they become removed. Form the vectors $\boldsymbol{n}=\left(n_{1}, \ldots, n_{I}\right)$ and $\boldsymbol{m}=\left(m_{1}, \ldots, m_{I}\right)$, and the matrix $\boldsymbol{\Gamma}=\left[\gamma_{i, j}\right]$. Let $\phi_{i}(\theta)=\mathrm{E}\left[\mathrm{e}^{-\theta T_{i}}\right]$, and, for $I$-vectors $\boldsymbol{x}$ and $\boldsymbol{y}$, let

$$
\left(\begin{array}{l}
\boldsymbol{x} \\
\boldsymbol{y}
\end{array}\right)=\prod_{i=1}^{I}\left(\begin{array}{l}
x_{i} \\
y_{i}
\end{array}\right) .
$$

Let $S_{i, j}$ represent the sum of the infectious periods of type- $j$ individuals, when $\boldsymbol{m}$ is the vector of $0 \mathrm{~s}$ that has a 1 in the $i$ th position. Form the vector $S_{i}=\left(S_{i, 1}, \ldots, S_{i, I}\right)$, and let $\psi_{i}(\boldsymbol{\theta})=$ $\mathrm{E}\left[\exp \left(-\boldsymbol{\theta} \cdot \boldsymbol{S}_{i}\right)\right]$.

Ball [1] showed that, for $\boldsymbol{\theta} \in[0, \infty)^{I}$,

$$
\psi_{i}(\boldsymbol{\theta})=\sum_{\boldsymbol{0} \leq \boldsymbol{w} \leq \boldsymbol{n}} g_{\boldsymbol{w}}(\boldsymbol{\theta})\left(\begin{array}{l}
\boldsymbol{n} \\
\boldsymbol{w}
\end{array}\right),
$$

where, for $\mathbf{0} \leq \boldsymbol{j} \leq \boldsymbol{n}$,

$$
\sum_{\mathbf{0} \leq \boldsymbol{w} \leq \boldsymbol{j}} \frac{g_{\boldsymbol{w}}(\boldsymbol{\theta})\left(\begin{array}{c}
j \\
w
\end{array}\right)}{\boldsymbol{\phi}\left(\boldsymbol{\theta}+(\boldsymbol{n}-\boldsymbol{j}) \Gamma^{T}\right)^{m+w}}=1
$$

where

$$
\boldsymbol{\phi}(\boldsymbol{\theta})^{\boldsymbol{x}}=\prod_{k=1}^{I} \phi_{k}\left(\theta_{k}\right)^{x_{k}} .
$$

This series of equations can be solved sequentially to give $\psi_{i}(\boldsymbol{\theta})$.

Now, to form the branching process of households, we let $K=I$, so that type- $k$ severity represents infectious pressure from a type- $i$ individual. For simplicity, we shall assume that all of the households have the same composition, so that there are $H=I$ initial configurations for a within-household epidemic, where, for $i=1, \ldots, I$, the initial infective is of type $i$. The probability of containment can now be obtained by solving (2).

Lefèvre and Picard [6] and Daley [4] gave an analogous result to Theorem 5 for SIR epidemics of this type. Consider two independent within-household SIR epidemics that differ only in their infectious period distributions. Let $T_{i}^{(j)}$ be a random variable with the type-i infectious period distribution for model $j \in\{1,2\}$. Let $S_{i}=\left(S_{i, 1}, \ldots, S_{i, I}\right)$ denote the household severity vector for a household with initial infective of type $i$. Lefèvre and Picard stated that if $T_{i}^{(1)} \geq_{\mathrm{Lt}} T_{i}^{(2)}$ for all $i=1, \ldots, I$ then $S_{i}^{(1)} \geq_{\mathrm{Lt}} \boldsymbol{S}_{i}^{(2)}$ for all $i$. When coupled with Theorem 5, this result implies that if $T_{i}^{(1)} \geq_{\mathrm{Lt}} T_{i}^{(2)}$ for all $i=1, \ldots, I$ then $\boldsymbol{G}_{i}^{(1)}(\boldsymbol{\alpha}) \leq \boldsymbol{G}_{i}(\boldsymbol{\alpha})$ for all $i$, i.e. the containment probability is smaller for model 1 than for model 2 .

This result is demonstrated in Figure 2 for the much simpler case where $I=1$ and all households are of size 4 . The infectious period distributions used in the figure all have mean 1 and satisfy

$$
1 \geq_{\mathrm{Lt}} U(0,2), \Gamma(2,2) \geq_{\mathrm{Lt}} \operatorname{Exp}(1) \geq_{\mathrm{Lt}} \Gamma\left(\frac{1}{2}, \frac{1}{2}\right),
$$

where $\Gamma(a, b)$ represents the gamma distribution with density $f_{X}(x)=b^{a} x^{a-1} \mathrm{e}^{-b x} / \Gamma(a)$ for $x>0$. Table 1 gives the corresponding mean number of infected individuals in a household and $R_{*}$ for epidemics with each infectious period distribution.

Note that the distributions $\Gamma(2,2)$ and $U(0,2)$ are not ordered under the Laplace transform ordering and the curves representing their containment probabilities cross. Also, note that, by Jensen's inequality, the constant infectious period distribution gives rise to the largest possible epidemic for a given mean infectious period length, i.e. $\mathrm{E}\left[\mathrm{e}^{-\theta T}\right] \leq \mathrm{e}^{-\theta \mathrm{E}[T]}$ for all $\theta \in[0, \infty)$. 


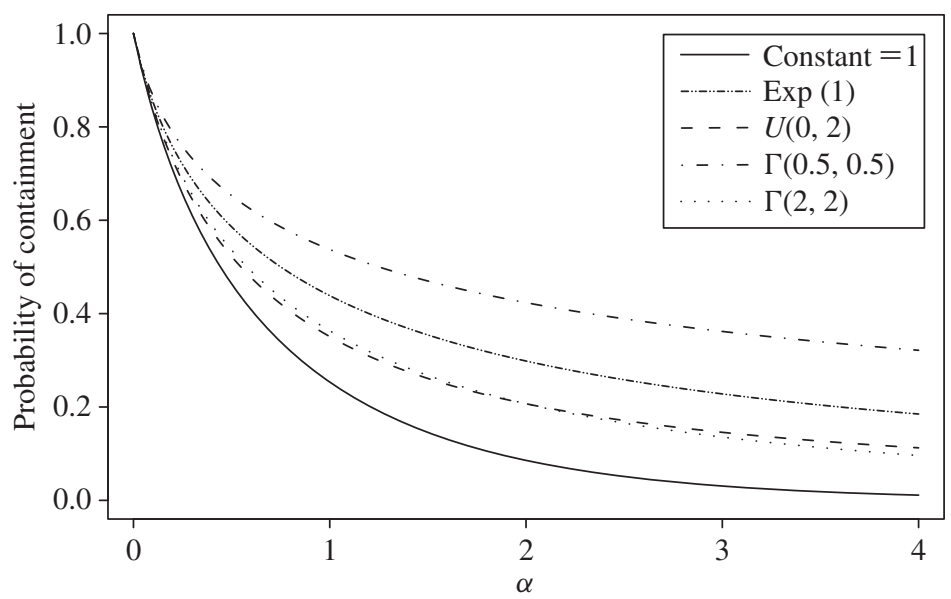

FIGURE 2: The containment probability, $G(\alpha)$, against the external contact rate, $\alpha$, when $\beta=0.4, \gamma=0.5$, $\mathrm{E}[T]=1$, and all households contain four individuals.

TABLE 1: Comparisons of the mean number of household infections and $R_{*}$ for five distributions of the infectious period, $T$, with $\mathrm{E}[T]=1$.

\begin{tabular}{ccc}
\hline Distribution of $T$ & Mean household infections & $R_{*}$ \\
\hline 1 & 1.78 & 1.11 \\
$\mathrm{U}(0,2)$ & 1.56 & 1.02 \\
$\Gamma(2,2)$ & 1.52 & 1.01 \\
$\operatorname{Exp}(1)$ & 1.34 & 0.94 \\
$\Gamma\left(\frac{1}{2}, \frac{1}{2}\right)$ & 1.11 & 0.84 \\
\hline
\end{tabular}

\subsection{Pandemic influenza}

Ferguson et al. [5] used household epidemic data to determine estimates of influenza natural history parameters and distributions. These estimates were then used to construct a large-scale simulation model of influenza transmission in rural Thailand, which was then used to test the effectiveness of proposed intervention strategies. In this section the modelling techniques discussed in this paper are used to construct an analogous model and calculate the probability of containment under different intervention strategies, providing an additional measure of intervention effectiveness.

We exactly replicate the within-household model of Ferguson et al. and the assumed effects of the interventions via simulation. However, the large-scale simulation model incorporates three levels of mixing: within-household, global, and workplace/school. We combine the latter two levels of mixing into the internal contact level, and to ensure the models remain comparable, the internal contact rate $\beta$ is chosen so that the reproduction number is 1.8 for both models.

5.3.1. Household model. An SEIR (susceptible-exposed-infective-removed) model is used to describe the within-household epidemic. Once infected, individuals experience an exposed period during which they are not infectious. This period has length distributed according to 
TABLE 2: The household size distribution of Thailand.

\begin{tabular}{cc}
\hline Household size & Proportion \\
\hline 1 & 0.150 \\
2 & 0.175 \\
3 & 0.230 \\
4 & 0.270 \\
5 & 0.165 \\
6 & 0.030 \\
7 & 0.015 \\
8 & 0.005 \\
9 & 0.004 \\
10 & 0.001 \\
\hline
\end{tabular}

0.5 days plus a Weibull distribution with power parameter 2.21 and scale parameter 1.1. It is assumed that $50 \%$ of infections are severe (strongly symptomatic) and these are twice as infectious as those that are nonsevere. In contrast to many other modelling papers, Ferguson et al. have a constant length infectious period during which infectiousness varies. The household severity is therefore defined to be the integral of the infectiousness level over the entire period of the within-household epidemic. It is sufficient to consider only this single type of severity $(K=1)$. Infectiousness for severe cases varies according to the density of a lognormal distribution with parameters -0.72 and 1.8 , truncated at 10 days and then renormalised; denote this density by $\kappa$. The probability of a household member avoiding infection in the first $t$ days of infectiousness from a severe case is assumed to be

$$
\exp \left(-\int_{0}^{t} \frac{0.94}{n^{0.8}} \kappa(u) \mathrm{d} u\right),
$$

where $n$ is the number of individuals in the household. The household size distribution is given in Table 2. Following Ferguson et al., the simulations were actually performed in a discretisation of the above model, in which all events that occur in $((t-1) / 4, t / 4]$ actually occur at time $t / 4$ for $t \in \mathbb{N}$. This does not affect the severity produced by the household, except by changing slightly the time at which the intervention (if there is one) is applied.

5.3.2. Intervention strategies. The following five intervention strategies are considered: no intervention, household isolation, household prophylaxis, blanket prohpylaxis, and social distancing.

Again following Ferguson et al. [5], severe infections exhibit healthcare-seeking behaviour 0.25 days after the start of the infectious period, which is assumed to coincide with the onset of symptoms. At this point the household interventions are triggered with probability 0.9 . In the household isolation intervention strategy it is assumed that no more internal or external contacts can emerge from a household after the intervention, and, therefore, any further withinhousehold severity is inconsequential. In the household prophylaxis intervention strategy, each individual is given a course of antivirals with probability 0.9 . It is assumed that a course of the antiviral drug Oseltamivir (Tamiflu) will reduce infectiousness by $60 \%$, reduce susceptibility by $30 \%$, and reduce the probability of a severe infection by $65 \%$. In the blanket prophylaxis intervention, each individual in the population is given a course of antivirals at the outset 


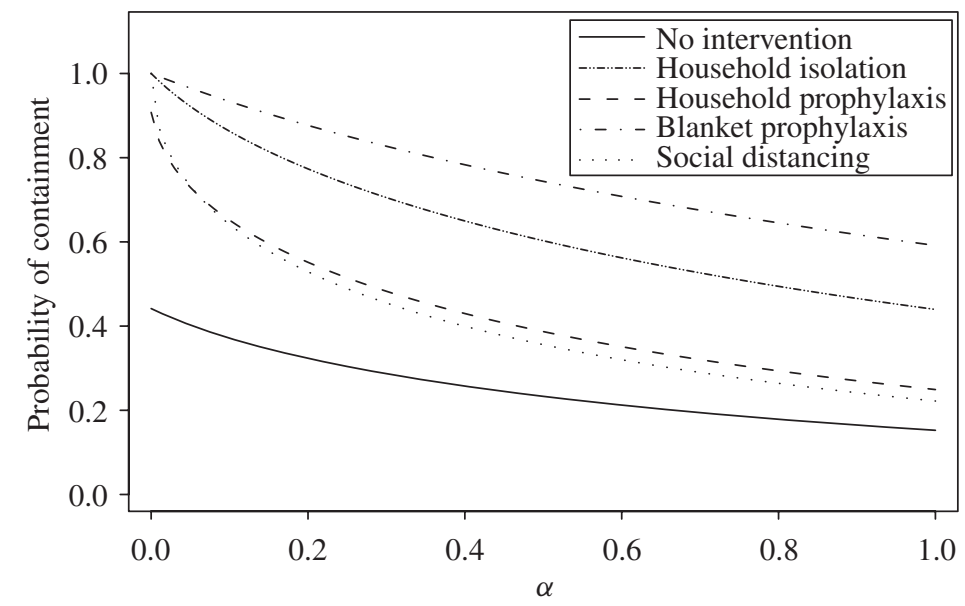

FIGURE 3: The probability of containment against the external contact rate, $\alpha$, for five intervention strategies with 10000 simulations of each of the 10 household sizes.

TABLE 3: The threshold parameter, $R_{*}$, after intervention for the five intervention strategies.

\begin{tabular}{lc}
\hline Intervention strategy & $R_{*}$ \\
\hline No intervention & 1.80 \\
Household isolation & 0.71 \\
Household prophylaxis & 1.05 \\
Blanket prophylaxis & 0.42 \\
Social distancing & 1.00 \\
\hline
\end{tabular}

with probability $90 \%$. Finally, in the social distancing intervention, the internal contact rate is decreased so that $R_{*}$ is reduced from 1.8 to the threshold level of 1 , at which the extinction of the epidemic becomes certain.

Figure 3 shows the probability of containment for the intervention strategies described above, and Table 3 gives the $R_{*}$ values. The household prophylaxis strategy and the social distancing strategy give similar improvements to the probability of containment. The fact that these are the least effective strategies is in broad agreement with the conclusions of Ferguson et al. [5], although our model is considerably simpler. It is interesting to note that, for some values of $\alpha$, household prophylaxis outperforms social distancing despite the fact that $R_{*}$ is lower for the social distancing strategy. This is due to the assumption that antivirals will reduce infectiousness; however, social distancing has not been assumed to affect the external contact rate.

The household isolation strategy is the best possible household-based intervention with this set of intervention trigger assumptions, as no contacts are assumed to be made after the intervention is triggered. Despite this, the blanket prophylaxis strategy has a substantially lower value of $R_{*}$. This substantial difference is not as strong in the containment probabilities due to the fact that approaching a containment probability of 1 becomes increasingly difficult. 


\section{Discussion}

An important feature of the model used in this paper is its flexibility. Very few assumptions are made concerning the within-household transmission of infection, and consequently, the model can be tailored to suit many different populations and diseases. This is demonstrated in Section 5.3, where a nonstandard SEIR model with variable infectiousness is used to model pandemic influenza and numerical results are obtained with little difficulty. The most restrictive model assumption is that there are only two levels of mixing within the population being modelled. A useful but challenging extension to this paper would be to consider further levels of mixing, for example workplaces and schools.

The mathematical results in this paper demonstrate that the containment probability can be viewed as a generalisation of the extinction probability of the branching process. However, the addition of external contacts to the branching process allows us not to consider separately the awkward special cases of singular and periodic processes.

The methods described in this paper can be used to compare and evaluate the effectiveness of intervention strategies for emerging pandemics before the outbreak of disease. This will become increasingly relevant and important as international surveillance improves and the early detection of emerging diseases becomes more commonplace.

\section{Acknowledgement}

Simon Spencer was supported by an EPSRC Doctoral Training Grant.

\section{References}

[1] BALL, F. (1986). A unified approach to the distribution of total size and total area under the trajectory of infectives in epidemic models. Adv. Appl. Prob. 18, 289-310.

[2] Ball, F., Mollison, D. and Scalia-Tomba, G. (1997). Epidemics with two levels of mixing. Ann. Appl. Prob. 7, 46-89.

[3] Ball, F. ANd Lyne, O. D. (2001). Stochastic multitype SIR epidemic among a population partitioned into households. Adv. Appl. Prob. 33, 99-123.

[4] Daley, D. J. (1990). The size of epidemics with variable infectious periods. Tech. Rep. SMS-012-90, Australian National University.

[5] Ferguson, N. M. et al. (2005). Strategies for containing an emerging influenza pandemic in Southeast Asia. Nature 437, 209-214.

[6] Lefèvre, C. P. AND PiCARD, P. (1993). An unusual stochastic order relation with some applications in sampling and epidemic theory. Adv. Appl. Prob. 25, 63-81.

[7] Longini, I. M. et al. (2005). Containing pandemic influenza at the source. Science 309, 1083-1087.

[8] Mardia, K. V., Kent, J. T. And Bibby, J. M. (1979). Multivariate Analysis. Academic Press, London.

[9] Mode, C. J. (1971). Multitype Branching Processes. Theory and Applications. American Elsevier, New York.

[10] Shaked, M. and Shanthikumar, J. G. (1994). Stochastic Orders and Their Applications. Academic Press, Boston, MA.

[11] Spencer, S. E. F. (2007). Stochastic epidemic models for emerging diseases. Doctoral Thesis, University of Nottingham. 